\title{
Relation between instability waves and low-frequency jet noise investigated with phased-microphone arrays
}

\author{
Takao Suzuki* \\ University of Fukui, Fukui, Fukui, 910-8507, Japan \\ Tim Colonius ${ }^{\dagger}$ \\ California Institute of Technology, Pasadena, CA, 91125, USA
}

\begin{abstract}
We study the relation between sound radiation patterns and the azimuthal-mode balance of instability-wave amplitude in a subsonic jet using mid-field and near-field phasedmicrophone arrays. To identify instability waves, we apply a least square optimization method, i.e. a beam-forming algorithm, to pressure signals obtained from a conical microphone array surrounding the jet from the nozzle exit to the end of the potential core. For the reference solutions, we use eigenfunctions based on linear stability analysis using the turbulent mean-velocity profiles and infer the amplitude of the axisymmetric and first two azimuthal modes associated with the Kelvin-Helmholtz instabilities. Likewise, pressure signals obtained from the mid-field array are decomposed into the azimuthal modes, and the directivity of low-frequency noise, particularly that of coherent sound, is analyzed. The azimuthal mode balances of the instability waves and acoustic waves are then compared, and the effects of compressibility, jet temperature and nozzle type (i.e. straight and chevron nozzles) are investigated. The results show that the directivity of low-frequency jet noise changes from the quadrupole radiation patterns to highly directive ones as increasing Mach number, while all instability modes are suppressed owing to compressibility without substantially varying the azimuthal-mode balance. The correlation of the azimuthal-mode balance between instability waves and coherent sound is stronger at higher Mach numbers. We also find that the radiation patterns of low-frequency noise change from quadrupoles to dipoles as the temperature increases at a low Mach number.
\end{abstract}

\section{Introduction}

$\mathrm{T}$ He present work is motivated by the need to better understand the sound radiated by large-scale coherent structures in round jets, including heated jets and jets with passive and active mixing devices at the nozzle lip (examples include tabs, ${ }^{1,2,3}$ chevrons, ${ }^{4}$ piezoelectric actuators, ${ }^{5}$ and microjets ${ }^{6}$ ). Large-scale structures in a jet are related to the Kelvin-Helmholtz instabilities of the time-averaged flow field. While this observation has lead to quantitative predictions of the near field evolution, ${ }^{7,8,9,10}$ the relation between the instability waves and the far-field sound has been a topic of debate. When large-scale structures are supersonically convected relative to the ambient, strong Mach waves are radiated and their amplitude levels can be directly calculated. ${ }^{11}$ In the subsonic case, some qualitative features of the sound generation can be derived from models based on linear stability analysis $7^{7,12,13}$ however, the amplitude of the sound radiation depends sensitively on the precise structure of the wave packet. ${ }^{13,14}$ Moreover, the relation between mixing devices and large-scale structures (i.e. instability waves) is unclear. These devices typically reduce jet noise

*Assistant Professor, Graduate School of Engineering. Member AIAA.

†Professor, Division of Engineering and Applied Science, Member AIAA. 
near the frequency of peak radiation but enhance high-frequency broadband noise. Yet, it is not presently understood if such noise reduction phenomena are related to the instability characteristics of the jet flow.

To quantitatively relate large-scale structures with jet noise generation, we must overcome the difficulty in measuring instability waves. Although several previous studies ${ }^{15,12,16,17}$ have successfully predicted pressure fluctuations in an acoustically excited jet based on linear stability analysis, identification of instability waves in unforced jets, particularly decomposition of azimuthal modes, requires special care for data analysis. Because these waves may coexist with convective disturbances (i.e. turbulence) and acoustic waves in the near field, there is no unique way to decompose them into different modes. One approach to overcome this problem is to introduce a phased-microphone array technique. Arndt et al. ${ }^{18}$ used a conical phased-microphone array and analyzed pressure fluctuations just outside the mixing layer based on the Proper Orthogonal Decomposition (POD). To extract the instability-wave components, we developed a beam-forming algorithm applying to pressure data from a conical phased-microphone array in the near-field (called a hydrodynamics array). Using eigenfunctions based on linear stability analysis, we successfully measured instability-wave amplitudes for the axisymmetric and first two azimuthal modes (i.e. $m=0, \pm 1$, and \pm 2 ). Excellent agreement obtained in this former study between the coherent pressure signals and the eigenfunctions demonstrates that instability waves predicted by the linear stability analysis very well represent near-field pressure fluctuations of turbulent jets from the exit to the end of the potential core at low frequencies. ${ }^{19}$

In the present study, we wish to extract acoustic-wave components that are associated with these instability waves and to decompose them into azimuthal modes. Ghosh et al. ${ }^{20}$ measured sound radiation patterns using a microphone array (not phased-array) and showed that each azimuthal mode exhibits strong directivity (i.e. lobe patterns) at low frequencies. Their results may suggest that low-frequency jet noise is associated with some organized flow structures. In fact, many source-detection studies ${ }^{21,22,23,24}$ have indicated that the low-frequency noise is generated near the end of the potential core, at which large-scale vortices collapse. Although some other previous studies ${ }^{25,26}$ have measured the azimuthal-mode distributions of the acoustic waves, sound pressure levels over a limited range of polar angles were not sufficiently informative to educe the role of large-scale flow structures on jet noise generation. This motivates us to introduce phase-microphone array techniques in order to extract acoustic waves associated with large-scale structures in a subsonic jet. In this study, we measure the azimuthal-mode balance of instability waves using a hydrodynamic array and compare it with the sound radiation patterns obtained from a mid-field array. In particular, we pay attention to coherent jet noise, hypothesizing that coherent acoustic signals are associated with large-scale structures. From eigenvectors of a cross spectral matrix, we extract the POD mode with the greatest eigenvalue for each azimuthal mode.

The outline of this paper is as follows: In the next section, we review the beam-forming algorithm detecting instability-wave amplitude and the POD analysis applying to the acoustic signals. We then describe the test conditions and the experimental facilities including the jet rig, and the near-field and mid-field microphone arrays. All these experiments were conducted at NASA Glenn Research Center over a wide range of Mach numbers and jet-temperature ratios as well as the nozzle types (i.e. straight versus chevron nozzles). Subsequently, the results of the instability-wave detection and the acoustic measurement have been analyzed, focusing on the effects of compressibility, the jet temperature and chevron nozzles, and the interpretation and implication are presented in the last section.

\section{Phased-array Processing}

\section{A. Identification of Instability Waves}

We summarize the instability-wave identification technique developed in Ref. 19 in this section. The basic idea is to consider the least square matching between phased-array pressure signals and the reference solution, thereby the algorithm being analogous to the beam-forming. For the reference solution, we use eigenfunctions based on linear stability analysis, where mean-velocity profiles were obtained by the Particle Image Velocimetry (PIV) in previous studies. ${ }^{27,28}$ Here, we define a cost function to be minimized as follows:

2 of 16

American Institute of Aeronautics and Astronautics 


$$
J\left(\omega, m, a_{s}\right) \equiv \sum_{l=1}^{N_{\text {mic }}}\left|\hat{\Pi}\left(\omega, m, a_{s} ; x_{l}, r_{l}, \theta_{l}\right)-\hat{\Theta}_{l}(\omega)\right|^{2},
$$

where $\omega$ denotes the angular frequency, $m$ the azimuthal mode number, $N_{\text {mic }}$ the number of microphones. $\hat{\Theta}_{l}$ represents the Fourier-transformed pressure signals at the $l^{\text {th }}$ microphone (to be precise, taken by the logarithmic scale ${ }^{19}$ ), and $\hat{\Pi}$ denotes the corresponding eigenfunction given by

$$
\hat{\Pi}\left(\omega, m, a_{s} ; x_{l}, r_{l}, \theta_{l}\right) \equiv a_{s} \bar{A}(\omega, m ; x, r) \exp \left[i \int_{0}^{x} k\left(x^{\prime}\right) d x^{\prime}\right]
$$

where $a_{s}$ denotes the complex amplitude, and $\bar{A}$ is normalized so that its maximum value is unity at each cross section. Assuming that the mean flow is locally parallel, the eigenfunctions are calculated at each cross section using a shooting method based on the axial mean-velocity and temperature profiles from PIV.

By differentiating $J$ with respect to $a_{s}$, we can determine the complex amplitude of the eigenfunction as

$$
\left(a_{s}\right)_{\min }(\omega, m)=\frac{\sum_{l=1}^{N_{\text {mic }}} \bar{A}^{*}\left(\omega, m ; x_{l}, r_{l}\right) \mathrm{e}^{-\operatorname{Im}[k] x_{l}} \mathrm{e}^{-\mathrm{i}\left(\operatorname{Re}[k] x_{l}+m \theta_{l}\right)} \hat{\Theta}_{l}(\omega)}{\sum_{l=1}^{N_{\text {mic }}}\left|\bar{A}\left(\omega, m ; x_{l}, r_{l}\right)\right|^{2} \mathrm{e}^{-2 \operatorname{Im}[k] x_{l}}} .
$$

Thus, both magnitude, $\left|\left(a_{s}\right)_{\min }\right|$, and phase, $\arg \left[\left(a_{s}\right)_{\min }\right]$, of the eigenfunction can be inferred for each $\omega$ and $m$. In this study, four periods of the target frequency are Fourier-transformed in the data processing, and the root mean square over 500 samples of $\left|a_{s}\right|$ is regarded as the instability-wave amplitude.

\section{B. Decomposition of Acoustic Signals}

To decompose the acoustic signals into the azimuthal modes, we first Fourier-transform the phased-array pressure data from the mid-field array (see figure 4 later) in time. Subsequently, we Fourier-transform them in the azimuthal direction at each ring; accordingly, the transformed pressure amplitude at the $l^{\text {th }}$ ring is denoted by $\hat{q}_{l}$. To adjust the distance from the noise source to the microphone position and to take into account the zenithal dependence, we define a vector generating the radiation pattern as

$$
\mathbf{q}\left(=q_{l}\right) \equiv \sqrt{\left(x_{l}-x_{s}\right)^{2}+r_{l}^{2}} \sqrt{\sin \theta_{l}} \hat{\mathbf{q}}_{l},
$$

where $\left(x_{l}, r_{l}\right)$ denotes the $l^{\text {th }}$ ring position, $\sin \theta_{l} \equiv r_{l} / \sqrt{\left(x_{l}-x_{s}\right)^{2}+r_{l}^{2}}$, and the source position is assumed to be $x_{s} / D=5$ in this study. A source-detection study using the same database ${ }^{29}$ showed that the primary noise sources are located approximately at $3<x / D<7$ at the frequency range of our interests, and this result is similar to other experimental studies. ${ }^{21,22,23,24}$ Hence, $q_{l} / \sqrt{\sin \theta_{l}}$ represents the sound directivity for each azimuthal mode at a given frequency. Moreover, since the zenithal intervals between the microphone rings are nearly equal in the array used for this study, the square norm, $|\mathbf{q}|^{2}$, corresponds to the sound intensity in the region covered by the array.

To extract coherent sound signals from the phased-array data, we perform the POD analysis in the frequency domain. With the transformed pressure signals defined in Eq. (4), we construct a cross spectral matrix of $N_{\text {ring }} \times N_{\text {ring }}$ as follows:

$$
\overline{\mathbf{q q}^{\dagger}} \text {, }
$$

where $N_{\text {ring }}$ denotes the number of microphone rings (i.e. $1 \leq l \leq N_{\text {ring }}$ ). Similar to the near-field data processing, four periods of the target frequency are Fourier-transformed to construct a cross spectral matrix, and they are averaged over 500 samples. Therefore, the eigenvectors of the cross spectral matrix are equivalent to the POD modes, and the one associated with the greatest eigenvalue represents the most energetic radiation pattern of the coherent sound. To compare its directivity with that of overall sound, we display the following quantity in polar plots later: 


$$
\sqrt{\lambda^{(1)}} u_{l}^{(1)} / \sqrt{\sin \theta_{l}}
$$

where $\lambda^{(1)}$ and $\mathbf{u}^{(1)}$ denote the greatest eigenvalue and the corresponding eigenvector, respectively.

\section{Experimental Facilities}

\section{A. SHJAR and Flow Conditions}

The experiments for both near-field and acoustic measurements were conducted using the Small Hot Jet Acoustic Rig (SHJAR) at NASA Glenn Research Center. A single-stream round jet was mounted $3.05 \mathrm{~m}$ from the ground in an anechoic dome with a $20 \mathrm{~m}$ radius. The jet exit was a converging nozzle, and the exit diameter was $5.08 \mathrm{~cm}(2.0 \mathrm{in})$. The wall of the dome was filled with foam wedges.

A variety of combinations of Mach numbers $\left(M_{\infty} \equiv U_{\text {jet }} / a_{\infty}=0.35\right.$ to 0.90$)$ and temperature ratios $\left(T_{\text {jet }} / T_{\infty}=0.84\right.$ to 2.70 ) as well as three nozzle types (see figure 1) were tested. The flow conditions were determined following the set points of previous experiments by Tanna ${ }^{30}$ (the flow characteristics in most of these cases are summarized in Refs. 27,28 ). The flow conditions considered in this study are listed in table 1. Mean-velocity deviations from the designated test conditions were within $1 \%$.

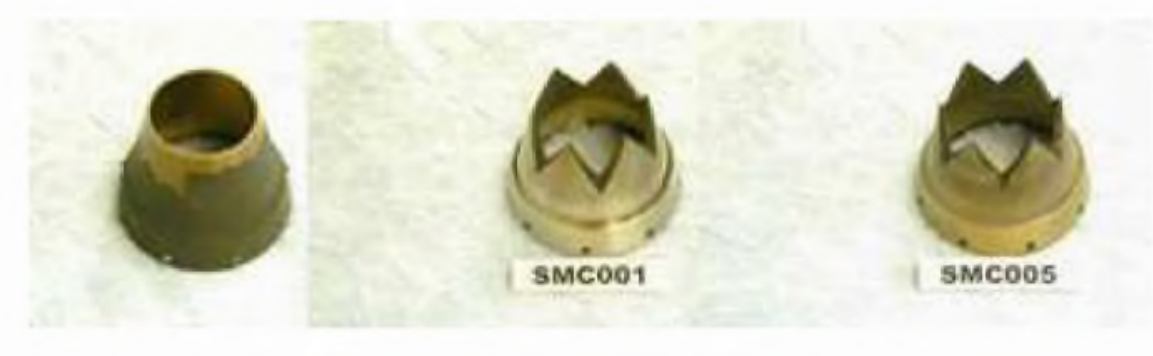

Figure 1. Photographs of the nozzles. Straight (SMC000), chevrons with $5^{\circ}$ penetration (SMC001), and chevrons with non-penetration (SMC005) from left to right.

Table 1. Operating conditions of jet flows. $M_{\text {jet }} \equiv(U / a)_{\text {jet }}$ and $\operatorname{Re} \equiv(\rho U D / \mu)_{\text {jet }}$.

\begin{tabular}{c|ccccc}
\hline Case & Set pt. & $M_{\infty}\left(M_{\text {jet }}\right)$ & $T_{\text {jet }} / T_{\infty}$ & Re & PIV flow data availability \\
\hline A & 1 & $0.35(0.35)$ & $0.98($ cold $)$ & $5 \times 10^{5}$ & No \\
B & 3 & $0.50(0.51)$ & $0.96($ cold $)$ & $7 \times 10^{5}$ & Yes \\
C & 5 & $0.70(0.74)$ & $0.91($ cold $)$ & $11 \times 10^{5}$ & No \\
D & 7 & $0.90(0.98)$ & $0.84($ cold $)$ & $16 \times 10^{5}$ & Yes \\
E & 23 & $0.50(0.38)$ & $1.76($ hot $)$ & $2 \times 10^{5}$ & Yes \\
F & 27 & $0.90(0.69)$ & $1.76($ hot $)$ & $4 \times 10^{5}$ & Yes \\
G & 42 & $0.50(0.31)$ & $2.70($ hot $)$ & $1 \times 10^{5}$ & No \\
H & 46 & $0.90(0.56)$ & $2.70($ hot $)$ & $2 \times 10^{5}$ & Yes
\end{tabular}

In this study, we select the target frequency to be $S t=0.35$ for unheated jets and $S t=0.30$ for heated ones. Ref. 19 demonstrated that the instability-wave detection method works best at these frequencies, but the characteristics of the sound radiation are similar in the range of $0.2 \leq S t \leq 0.6$. 


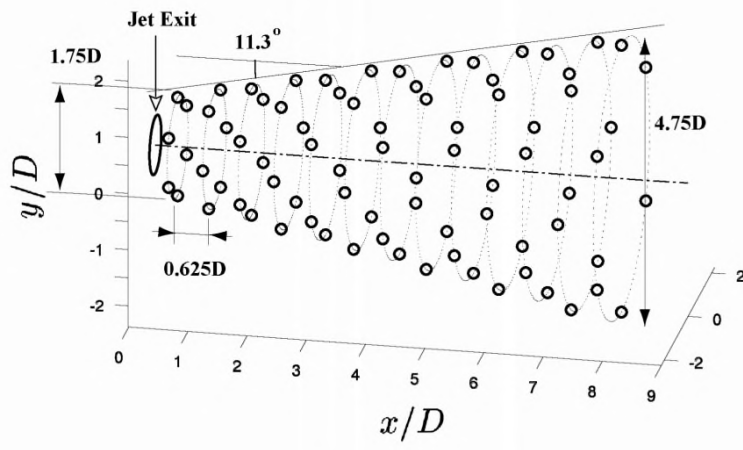

Figure 2. Microphone distribution of the hydrodynamic array. The center of the jet exit is taken to be the origin.

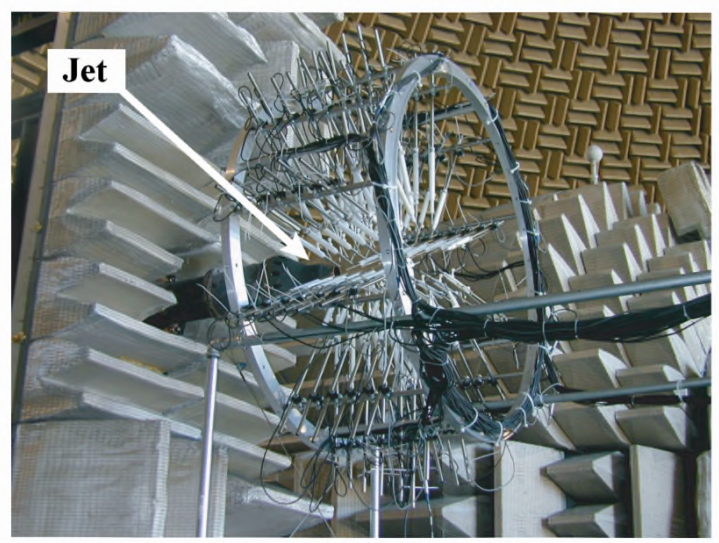

Figure 3. Photograph of the hydrodynamic array with the SHJAR facility.

\section{B. Mean-flow Data from PIV}

To construct the eigenfunctions of linear stability analysis, we use mean-flow data measured by the group directed by Bridges at NASA Glenn Research Center. ${ }^{27,28}$ Velocity fields on cross-sectional and streamwise planes were obtained using PIV with resolutions of approximately $0.1 D$ and $0.02 D$ in the streamwise and the radial directions, respectively. Although the mean-flow fields for cases $\mathrm{A}, \mathrm{C}$ and $\mathrm{G}$ are not available, they are estimated from case $\mathrm{B}$, the average of cases $\mathrm{B}$ and $\mathrm{D}$, and case $\mathrm{H}$, respectively, by scaling with the free-stream jet velocity ignoring compressibility.

To smoothly integrate eigenfunctions, we fit the axial mean-velocity data with a hyperbolic tangent profile, $U_{0}(r) \approx \frac{U_{\max }}{2}\left(\tanh \left[s\left(r+r_{0}\right)\right]-\tanh \left[s\left(r-r_{0}\right)\right]\right)$ : These three parameters, $U_{\max }, s$, and $r_{0}$, are optimized in a least-square sense at each cross section. From the axial velocity profiles, temperature profiles are estimated using the Crocco-Busemann relation. The accuracy of this treatment is described in Ref. 19.

Since the mean-velocity profile of the non-penetration chevron nozzle (SMC005) is nearly axisymmetric, we perform linear stability analysis with the velocity field averaged in the azimuthal direction and determine the instability-wave amplitude. For the high-penetration chevrons (SMC001), we do not apply the instabilitywave detection technique because of severe mean-flow distortion.

\section{Hydrodynamic Array}

The hydrodynamic array consists of 13 rings with 6 microphones each, for a total of 78 quarter-inch microphones (see figure 2 for the microphone distribution). The rings are equally spaced in the streamwise direction, and the azimuthal angles of the microphones on each ring are staggered every other ring. The spreading angle of the array (the half angle of the cone) is set to be $11.3^{\circ}$. Each microphone is supported by a non-conductive plastic sleeve, and it is joined to a stinger mounted on a cylindrical steel frame measuring $1.22 \mathrm{~m}$ diameter and $0.42 \mathrm{~m}$ axial length (see figure 3 for a photograph of the hydrodynamic array).

The whole array is mounted on a structure with wheels so that the effective radial position of the microphones is varied by shifting the axial position and reselecting the rings used for the beam-forming process (seven rings are used to generate a cross spectral matrix at each operating condition). The radius at the center of the array is then varied in the range of $1.0 \leq r / D \leq 1.75$ (at $x / D=2.25$ ). Thus, the radial decay of pressure signals is compared with the eigenfunction at each operating condition, and the feasible radial position is evaluated. Note that when the array is shifted upstream to measure pressure signals at a larger radius, first few upstream rings are removed. 


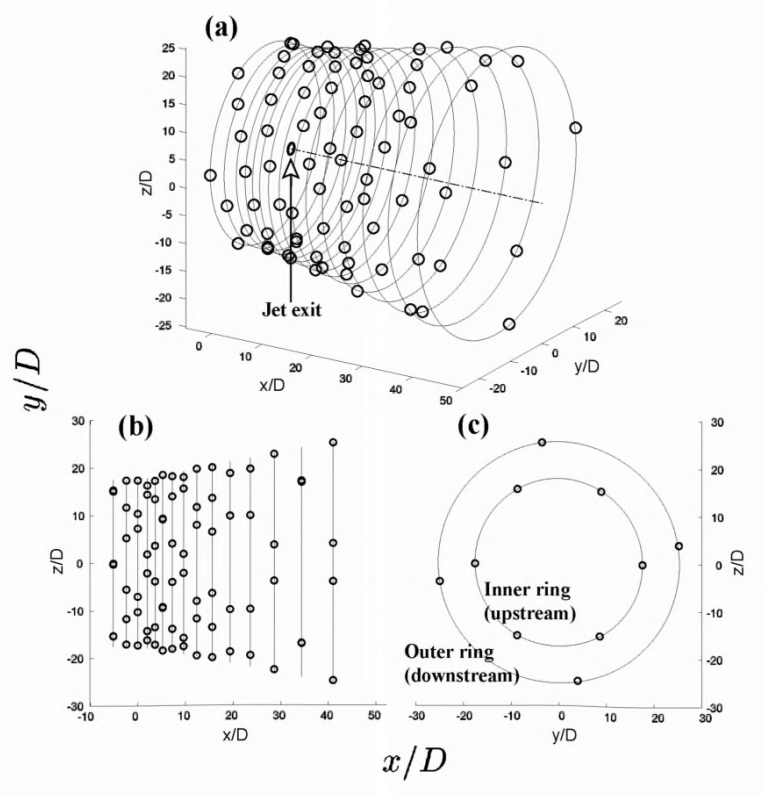

Figure 4. Microphone distribution of the midfield array. (a) three dimensional view. (b) side view. (c) front view.

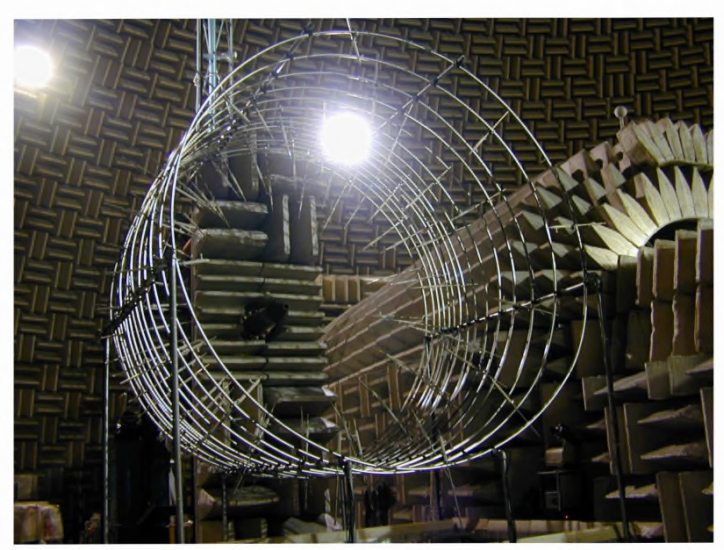

Figure 5. Photograph of the mid-field array with the SHJAR facility.

\section{Mid-field Array}

The mid-field microphone array for the acoustic measurement has 15 rings, the first 10 of which (upstream) have six microphones, and the latter 5 of which (downstream) have four microphones, all axisymmetrically distributed (see figure 4 for the microphone distribution and figure 5 for a photograph). Thus, it consists of a total of 80 quarter-inch microphones. The spreading angle of the array is approximately $11^{\circ}$, and the array covers $31.7^{\circ}<\theta<106.3^{\circ}$ in the polar angle, centered at the nozzle exit (it covers $35.1^{\circ}<\theta<119.9^{\circ}$ if the center is taken at $x / D=5$ ). The axial ring positions were designed so that the polar angles are about equally spaced. The distances from the jet exit to the microphones are in the range of $17.3 D<\sqrt{x^{2}+r^{2}}<48.3 D$, while the ambient wave-length is, for example, $\lambda=5.7 D$ at $S t=0.35$ for $M_{\infty}=0.5$.

We should note that compared to the hydrodynamic array, the aliasing error in the azimuthal direction can be more significant in the mid-field array, particular for the $m=2$ mode. Unlike near-field fluctuations, the energy contained in higher azimuthal modes is relatively large in acoustic signals. This issue is most severe at the five downstream rings, at which only four microphones are distributed in the azimuthal direction. For example, when the azimuthal spacings of the four microphones are exactly equal, the amplitudes of the $m= \pm 2$ modes become identical. By reducing the number of the microphones from six to four at the rest of the ten rings, the amplitude of the $m=2$ mode is increased by $20-30 \%$ on average. Therefore, the acoustic intensity of $m=2$ is overemphasized at shallow angles. In this study, the acoustic data are analyzed by comparing the change between different flow conditions.

\section{Results and Discussion}

\section{A. Effects of Compressibility}

First, we study the compressibility effects for unheated jets. Figures $6-8$ show the evolution of instability waves (on the left) and the sound radiation patterns (on the right) for the axisymmetric and first two azimuthal modes at three different Mach numbers. Here, we display only positive modes (i.e. $m=+1$ and 

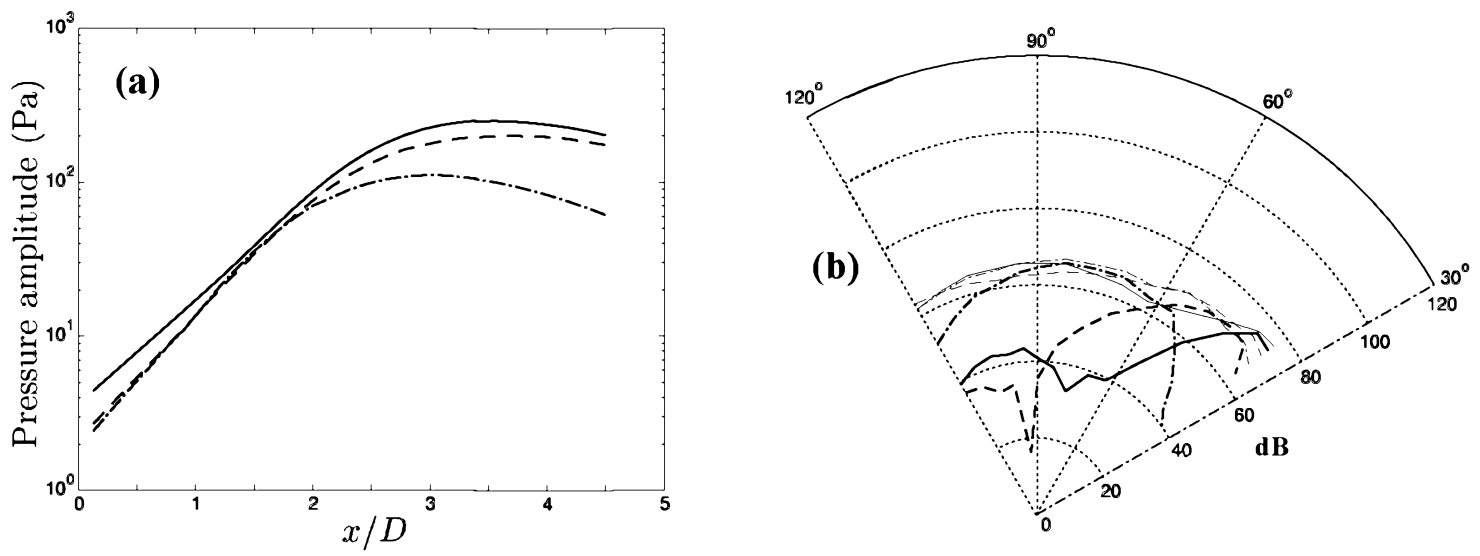

Figure 6. Streamwise evolution of eigenfunctions and radiation patterns of acoustic waves at $M_{\infty}=0.5$ for an unheated jet $(S t=0.35)$. Line patterns:,$- m=0 ;---, m=1 ;-\cdots-, m=2$. (a) Pressure amplitudes inferred from the beam-forming are plotted along $r / D=0.5$ (unit is pascal). (b) Pressure amplitudes (thinner lines) and the first eigenmodes of the cross spectral matrices (thicker lines) are depicted in a polar plot. Amplitude at $\sqrt{(x-5 D)^{2}+r^{2}}=30 D$ is calculated for each mode and plotted on a $\mathrm{dB}$ scale.
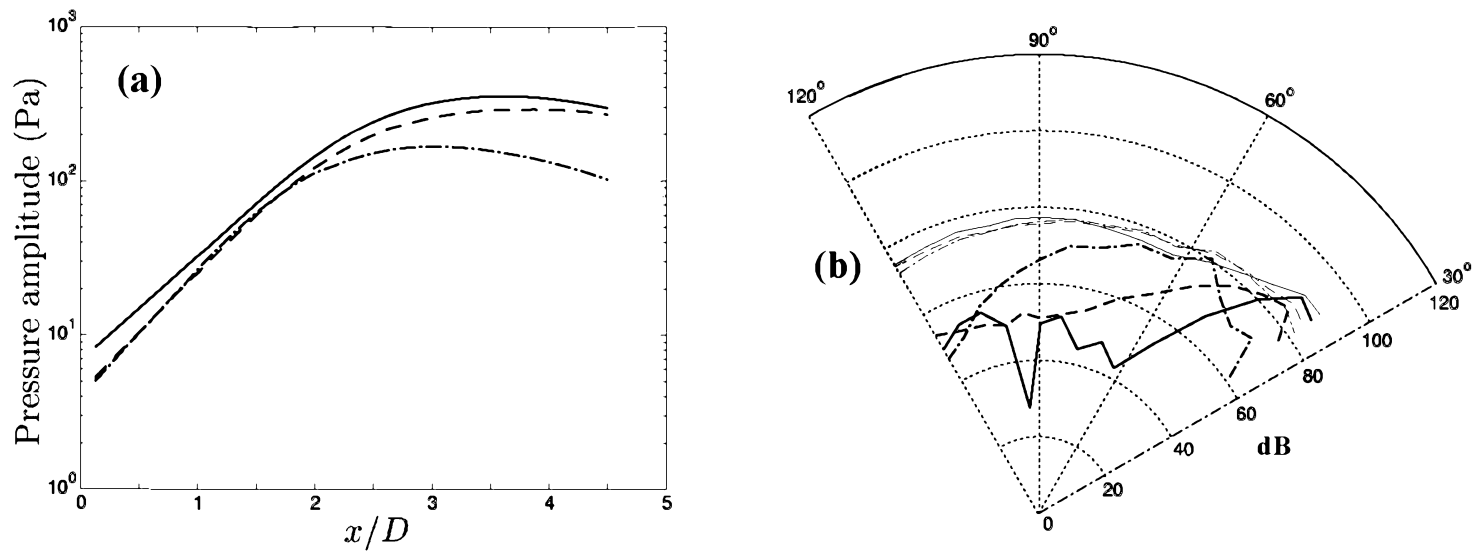

Figure 7. Streamwise evolution of eigenfunctions and radiation patterns of acoustic waves at $M_{\infty}=0.7$ for an unheated jet. Notation is the same as figure 6.
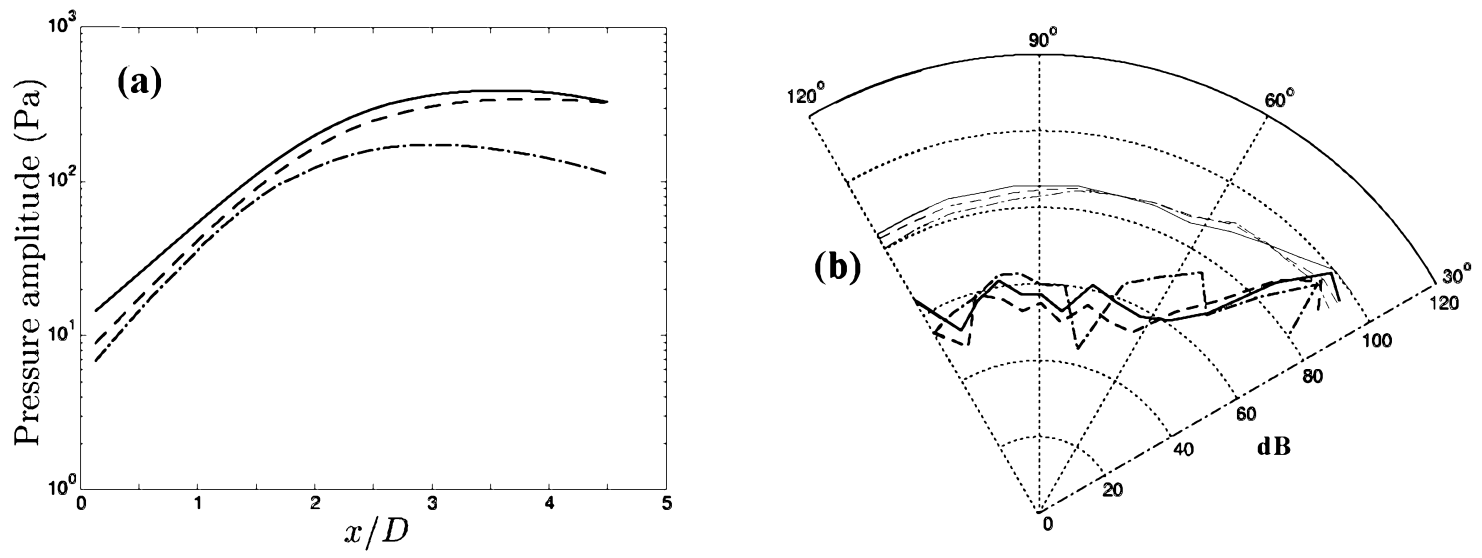

Figure 8. Streamwise evolution of eigenfunctions and radiation patterns of acoustic waves at $M_{\infty}=0.9$ for an unheated jet. Notation is the same as figure 6 . 
+2) for the azimuthal modes, but their negative ones are statistically the same. As the Mach number is increased, the growth rates of all the modes are suppressed; accordingly, instability waves are saturated farther downstream owing to compressibility, but their mode balances are quite similar. To be precise, their initial pressure disturbances are proportional to $M_{\infty}^{2}$, and they are amplified by the Kelvin-Helmholtz instability in the streamwise direction (refer to Ref. 19 for details).

In contrast, the radiation patterns substantially change with increasing Mach number. At the low Mach number $\left(M_{\infty}=0.5\right.$, figure 6$)$, the coherent signals depict clear lobe patterns, and they are qualitatively similar to the quadrupole directivity for each azimuthal mode (see figure 9 (a) for the quadrupole radiation patterns). For example, the $m=0$ mode of overall sound exhibits two lobes (the null is approximately $60^{\circ}$ and the third lobe may be hidden at higher angles), and its POD mode mainly captures the downstream lobe. These trends are consistent with the results of another study using the same database. ${ }^{29}$ As the Mach number increases, the intensity, particularly that of coherent sound, is concentrated at shallower angles. This may be caused by refraction, although expected lobe patterns cannot be observed near $90^{\circ}$, at which the refraction effect is negligible.

Figure 10 compares the azimuthal-mode balance of the instability waves (on the left) and the acoustic waves (on the right). For the instability waves, the peak pressure amplitude along the mixing layer is displayed, while for the acoustic waves, crude estimates of the squared intensity, $|\mathbf{q}|$ in Eq. (4), for the total pressure and the first POD mode, given by Eq. (6), are overlaid. The mode balance of the primary coherent sound becomes somewhat similar to that of the instability waves with increasing Mach number, while the rest of the acoustic parts is nearly independent of it. This may indicate that at low Mach numbers, jet noise is primarily generated with the non-linear process, which may be well represented by quadrupole-type source terms. As the Mach number is increased, coherent sound is substantially influenced by large-scale structures. This is consistent with the fact that the phase velocity of instability waves approaches the sonic speed at higher Mach numbers.

Note that the instability amplitudes, which are scaled by $M_{\infty}^{2}$, show clear suppression with increasing Mach number. In contrast, we cannot observe such reduction in acoustic amplitudes normalized by $M_{\infty}^{4}$, referring to Lighthill's acoustic analogy. ${ }^{31}$ We should remember that the array covers a limited range of polar angles; therefore, much acoustic intensity at higher angles radiated from lower Mach-number jets is excluded in these results.

\section{B. Effects of Jet Temperature}

Next, we study the temperature effects. Figures $11-13$ similarly show the evolution of instability waves and the sound radiation patterns at three different temperatures. As predicted by linear stability analysis, the amplification of the $m=0$ mode is weaker than the $m=1$ mode for an unheated jet, and the sound levels for these two modes are comparable.

As the jet is heated $\left(T_{\text {jet }} / T_{\infty}=1.76\right)$, instability waves are saturated closer to the nozzle exit, and the axisymmetric mode is greatly enhanced compared with other two modes (see figure 12). The enhancernent of the $m=0$ mode can also be seen in the sound radiation patterns. However, it should be noticed that the lobe patterns of the sound are changed from the unheated jet in figure 11. Particularly for $m=1$, the downstream lobe of the POD mode is shifted toward higher angles as the jet is heated. Moreover, the upstrearn lobe of the overall sound for $m=0$ in the unheated jet disappears in the heated jet. These trends become more noticeable as we further increase the jet temperature $\left(T_{\text {jet }} / T_{\infty}=2.70\right)$. Figure 13 shows that the downstrearn lobe is further enhanced for $m=0$, and the POD mode for $m=1$ is peaked nearly at $\theta=90^{\circ}$. These two modes are substantially amplified in the acoustic waves, while the instability-wave amplitude for $m=1$ is varied only slightly.

Many studies (e.g. Refs. 32,33) have discussed the presence of additional acoustic sources in heated jets, owing to significant fluctuations in density, and they have been found to display a dipole directivity. In our study, the lobe patterns of the coherent sound observed in the heated jets are qualitatively similar to the dipole radiation for $m=0$ and $m=1$ (see figure $9(\mathrm{~b})$ for the dipole directivity) and seern to support the

\section{8 of 16}

American Institute of Aeronautics and Astronautics 

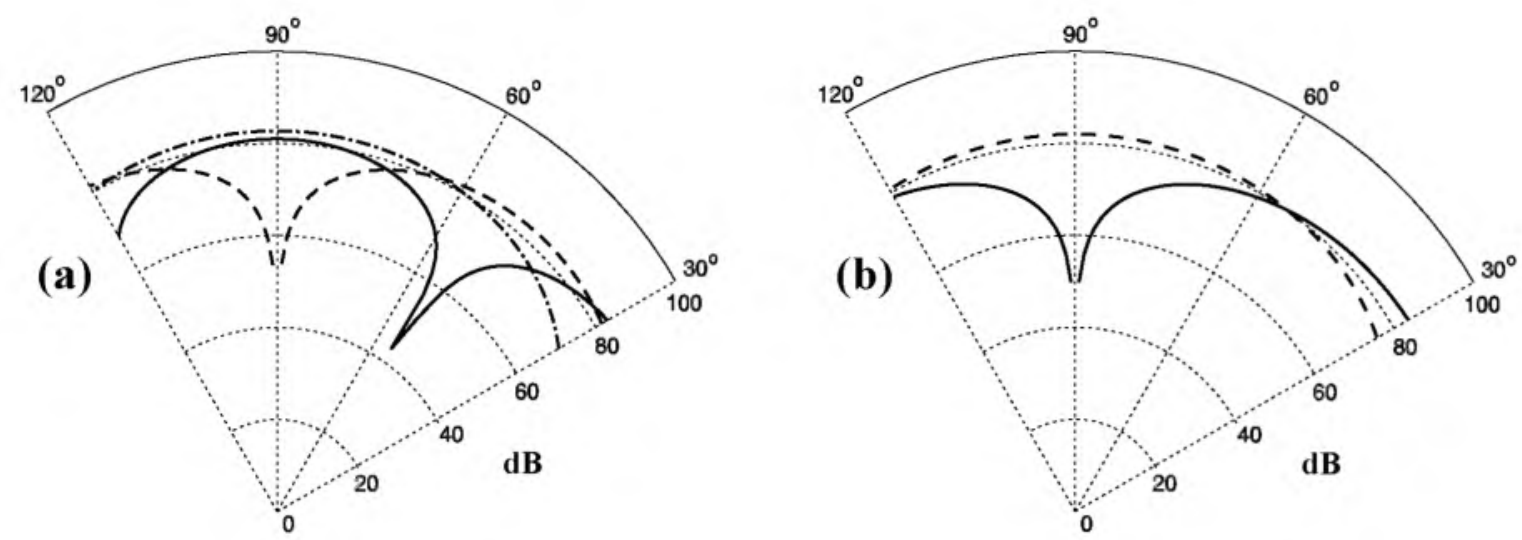

Figure 9. Directivity of spherical multipoles: (a) quadrupoles; (b) dipoles. Line patterns:,,$- m=0$; --- , $m=1 ;-\cdot-\cdot-, m=2$ for each zenithal mode. Magnitudes are arbitrary scaled.
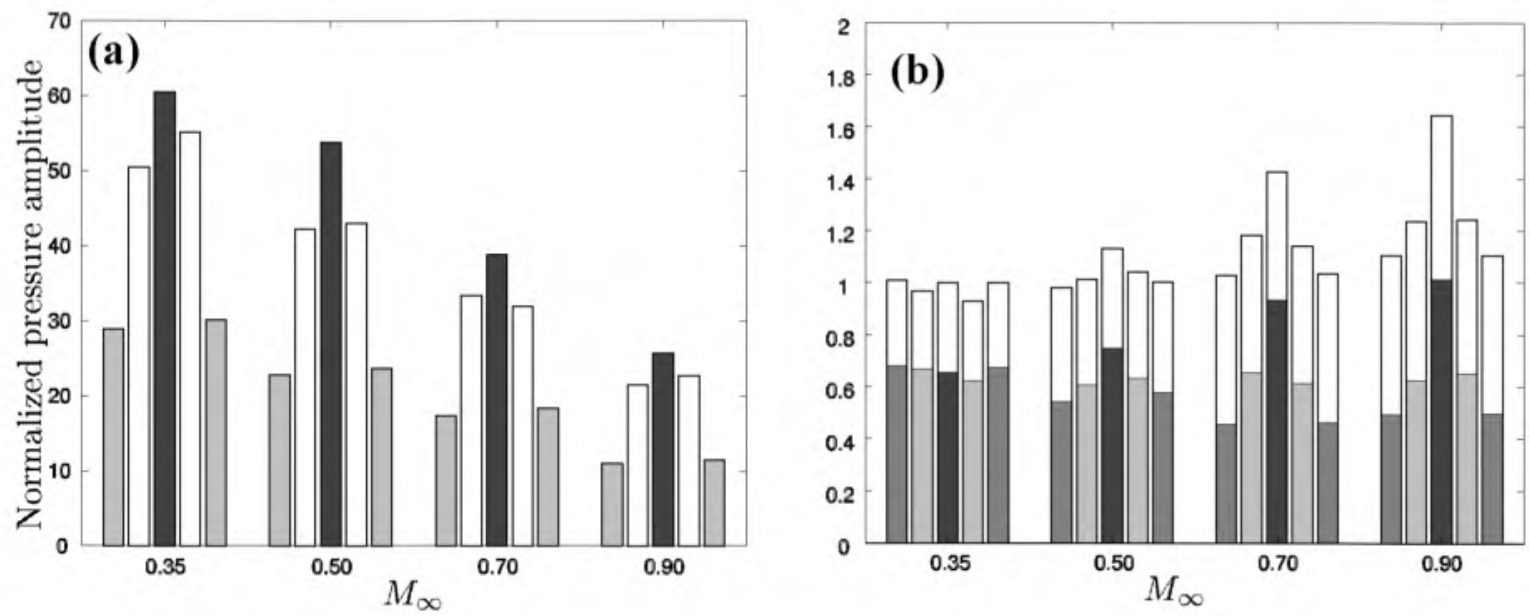

Figure 10. Comparison of the azimuthal mode balance at $S t=0.35$ for unheated jets. Each cluster shows amplitudes of the $m=-2,-1,0,1,2$ modes from left to right. (a) Instability-wave balance (the peak amplitude along $r / D=0.5$ ). Amplitudes are scaled by $M_{\infty}^{2}$ (amplitude of $m=0$ at the nozzle exit is taken as unity). (b) Acoustic mode balance. White bars denote the overall sound, and colored ones denote the first POD modes. Amplitudes are scaled by $M_{\infty}^{4}$, and they are normalized by the overall sound of the $m=0$ mode at $M_{\infty}=0.35$.

hypothesis that the coherent sound changes from the quadrupole-type to the dipole-type with increasing jet temperature.

Figure 14 compares the azimuthal-mode balance of the instability waves and the acoustic waves. In the instability-wave balance, only the axisymmetric mode is greatly amplified. In contrast, both $m=0$ and $m=1$ modes are enhanced in the acoustic waves although the enhancement of the axisymmetric mode is more pronounced. This may be explained from the fact that the $m=0$ and $m=1$ modes include dipoles, but the leading order for $m=2$ is only a quadrupole.

Figures 15-17 display the comparison between instability waves and acoustic waves at a higher Mach number $\left(M_{\infty}=0.9\right)$. Although the compressibility effect suppresses the growth rates of instability waves at a higher Mach number, the rest of the instability-wave characteristics are relatively similar to those at the lower Mach number; namely, only the axisymmetric mode is enhanced and all modes are saturated closer to the nozzle exit as the temperature ratio is increased. In contrast, sound directivity seems to be substantially influenced by the refraction effect, and the downstream lobe for $m=0$ becomes dominant at 

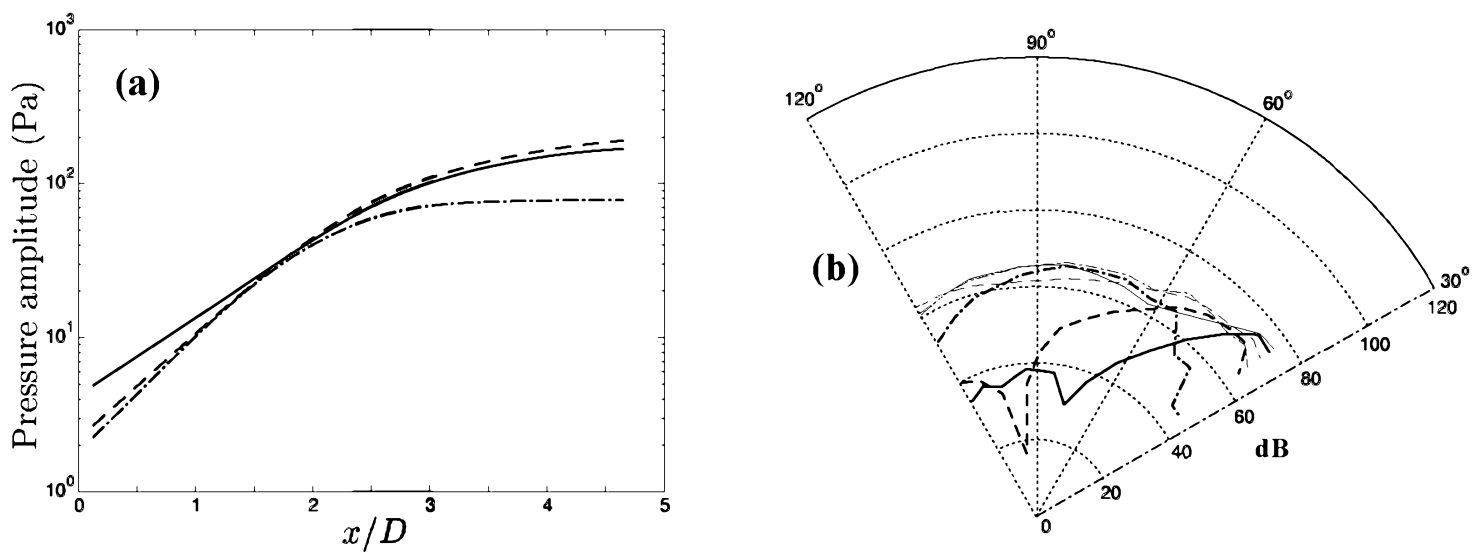

Figure 11. Streamwise evolution of eigenfunctions and radiation patterns of acoustic waves at $M_{\infty}=0.5$ for unheated jets $(S t=0.30)$. Notation is the same as figure 6. (a) Pressure amplitudes inferred from the beamforming (unit is pascal). (b) Pressure amplitudes (thinner lines) and the first eigenmodes of the cross spectral matrices (thicker lines).
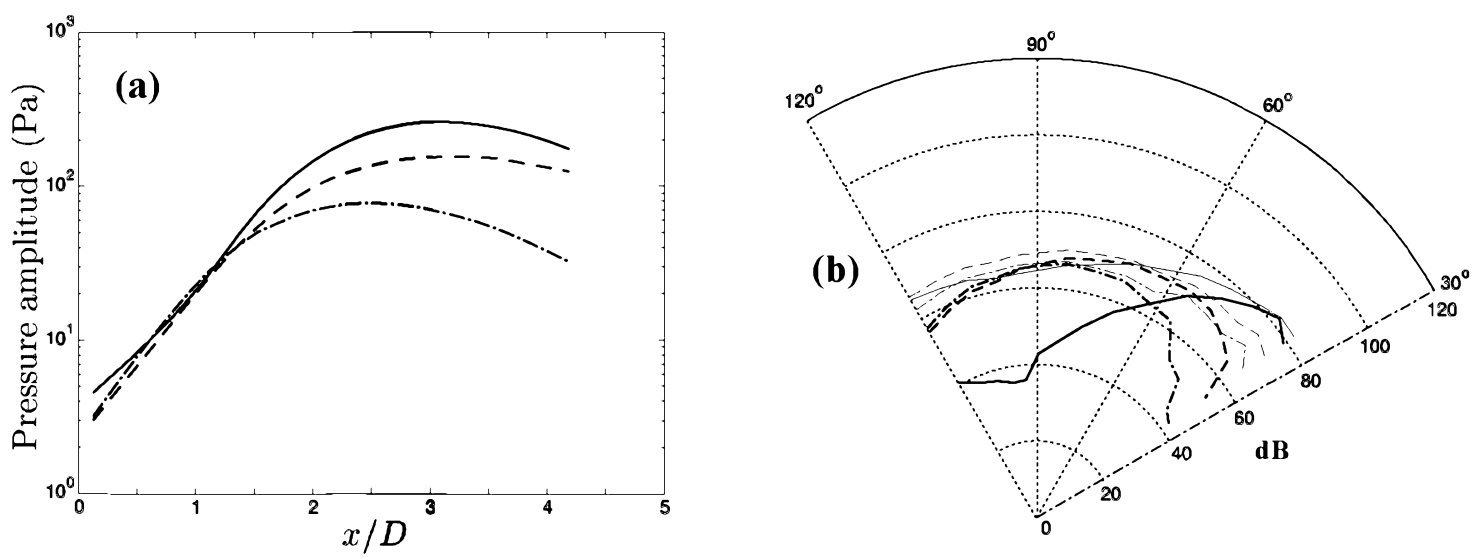

Figure 12. Streamwise evolution of eigenfunctions and radiation patterns of acoustic waves at $M_{\infty}=0.5$ for $T_{\text {jet }} / T_{\infty}=1.76$. Notation is the same as figure 6.
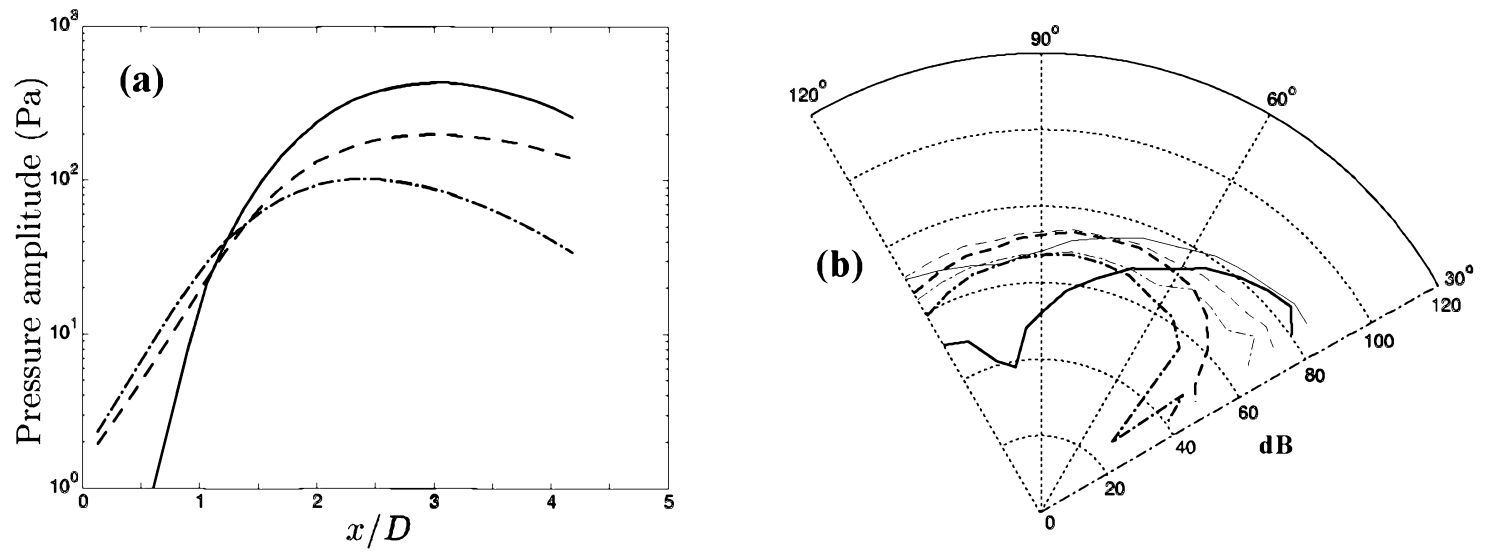

Figure 13. Streamwise evolution of eigenfunctions and radiation patterns of acoustic waves at $M_{\infty}=0.5$ for $T_{\text {jet }} / T_{\infty}=2.70$. Notation is the same as figure 6 .

10 of 16 

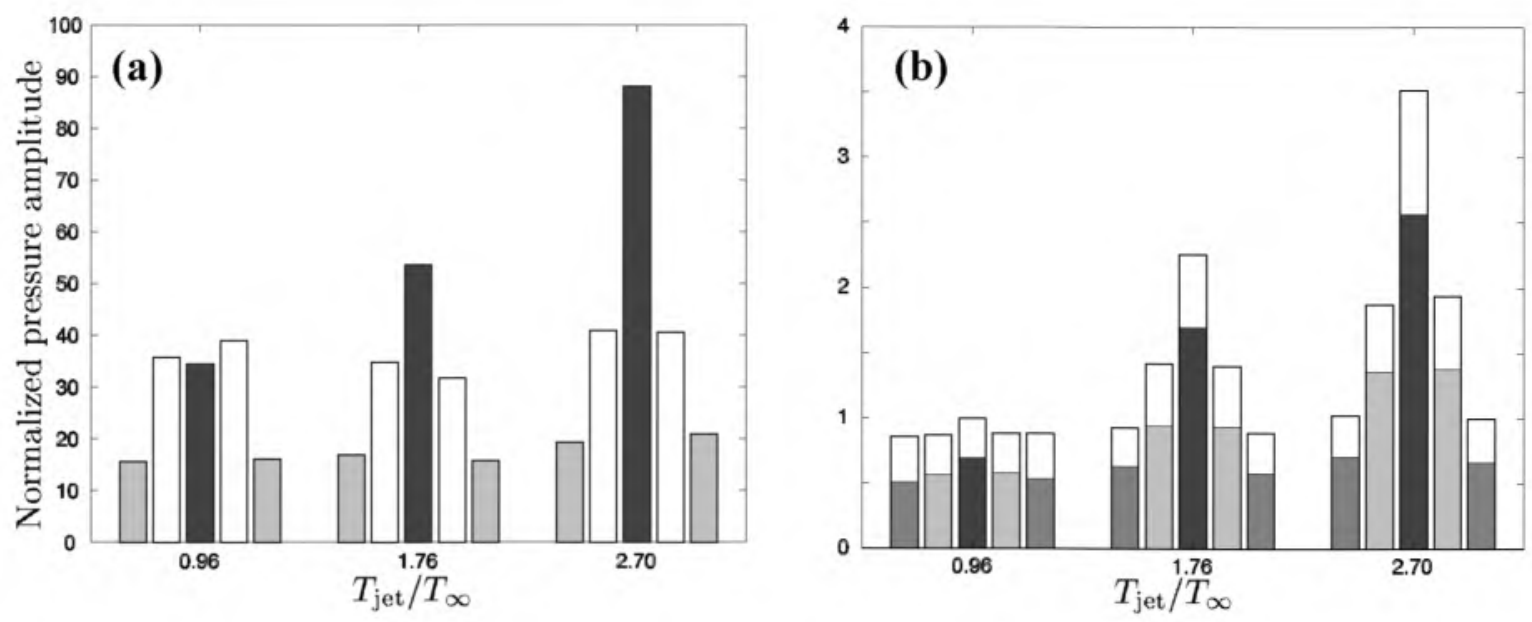

Figure 14. Comparison of the azimuthal mode balance at $M_{\infty}=0.5$ for $S t=0.30$. Each cluster shows amplitudes of the $m=-2,-1,0,1,2$ modes from left to right. (a) Instability-wave balance (the peak amplitude along $r / D=0.5$ ). Amplitudes are normalized by that of $m=0$ at the nozzle exit. (b) Acoustic mode balance. White bars denote the overall sound, and colored ones denote the first POD modes. Amplitudes are normalized by the overall sound of the unheated case for $m=0$.

all temperatures. Moreover, the overall sound levels for the two azimuthal modes are nearly comparable. As mentioned above, the density variation across the mixing layer may cause to generate dipole-type radiation, but its magnitude relative to the velocity fluctuations becomes smaller with increasing Mach number. As a result, the sound radiation patterns are less likely affected by the temperature increase at $M_{\infty}=0.9$.

The variation in the azimuthal-mode balance at $M_{\infty}=0.9$ can be clearly seen in figure 18 . Only the axisymmetric mode is significantly enhanced in the instability waves, and that of the acoustic waves is accordingly amplified. The rate of amplification in acoustic waves from the unheated jet to $T_{\text {jet }} / T_{\infty}=2.70$ is yet about $50 \%$, and this is much smaller than that at $M_{\infty}=0.5$ (refer to figure 14). The azimuthal modes, $m=1$ and $m=2$, are nearly unchanged for both near- and far-fields at $M_{\infty}=0.9$. Thus, the trends of instability waves and the sound become closer at the higher Mach number, and the shift to a dipole pattern observed at the lower Mach number appears to be less significant at the higher Mach number.

\section{Effects of Chevron Nozzles}

Finally, we consider the effects of chevron nozzles. Figures $19-21$ compare the instability-wave evolution and the acoustic radiation patterns for the three different nozzles. In the non-penetration chevron nozzle (SMC005), instability waves are saturated closer to the nozzle exit compared with the straight nozzle (SMC000) owing to the mixing enhancement. Moreover, amplitudes of all the modes are enhanced probably because the effective diameter of the jet is increased. Although amplitudes of instability waves are not measured for the high-penetration chevrons (SMC001) owing to the significant mean-flow distortion, the amplitude levels of raw pressure fluctuations are observed to be still slightly higher than those of SMC005 (not shown). On the other hand, the sound radiation patterns are almost the same for all nozzles. Although the structures of the instability waves are significantly altered by chevrons, variation in acoustic waves is very subtle.

The comparison of the azimuthal-mode balance demonstrates these trends more clearly. Figure 22 shows that all the instability waves are almost equally amplified by the chevrons, while there is little change in both overall sound and coherent one. The same trend is observed over a wide range of frequencies (at least, $0.1<S t<0.5$ ). Thus, the noise reduction with chevron nozzles is not originated with suppression of instability-wave amplitude. 

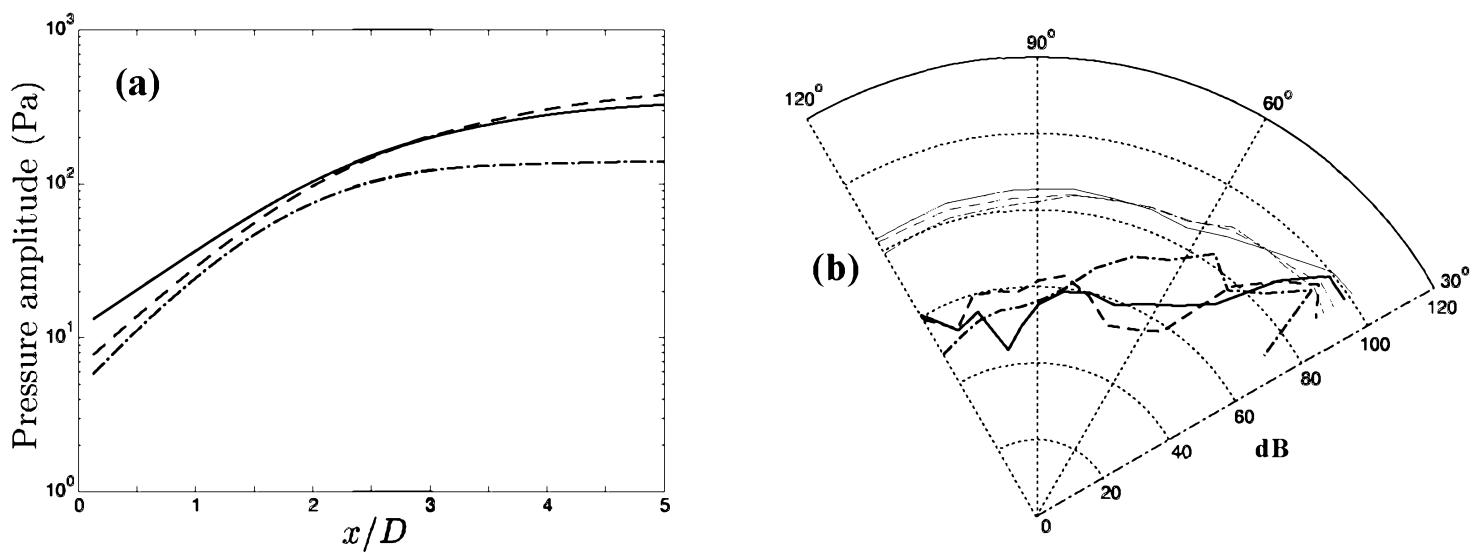

Figure 15. Streamwise evolution of eigenfunctions and radiation patterns of acoustic waves at $M_{\infty}=0.9$ for unheated jets $(S t=0.30)$. Notation is the same as figure 6. (a) Pressure amplitudes inferred from the beamforming (unit is pascal). (b) Pressure amplitudes (thinner lines) and the first eigenmodes of the cross spectral matrices (thicker lines).
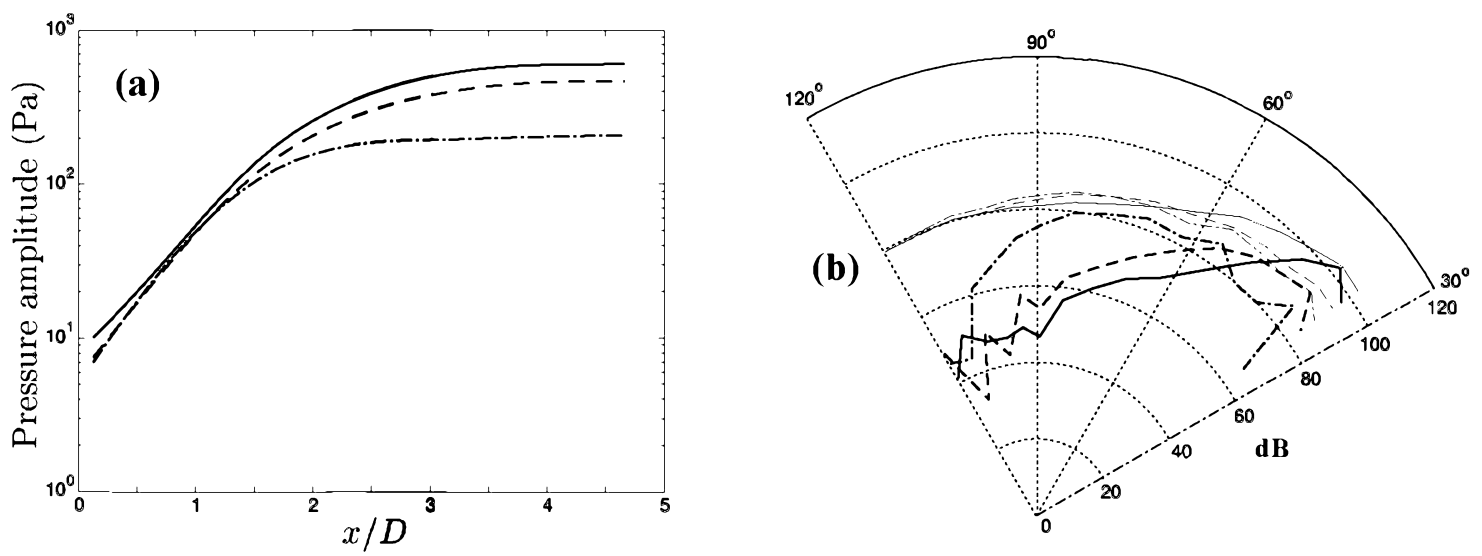

Figure 16. Streamwise evolution of eigenfunctions and radiation patterns of acoustic waves at $M_{\infty}=0.9$ for $T_{\text {jet }} / T_{\infty}=1.76$. Notation is the same as figure 6.
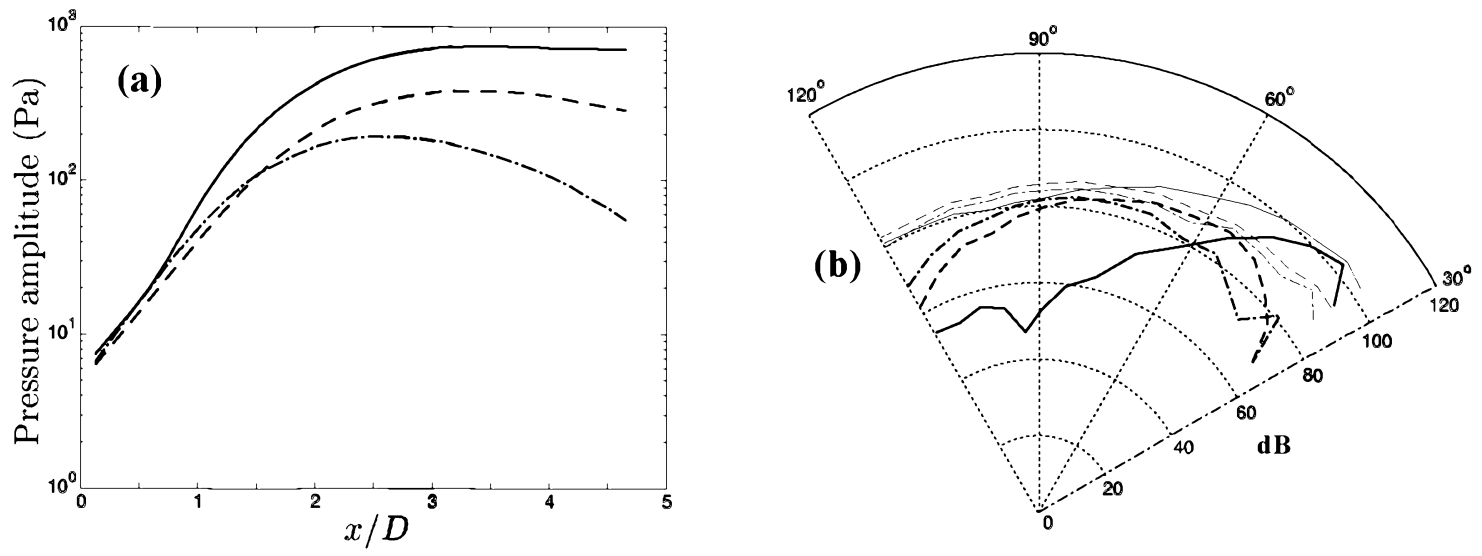

Figure 17. Streamwise evolution of eigenfunctions and radiation patterns of acoustic waves at $M_{\infty}=0.9$ for $T_{\text {jet }} / T_{\infty}=2.70$. Notation is the same as figure 6 .

12 of 16 

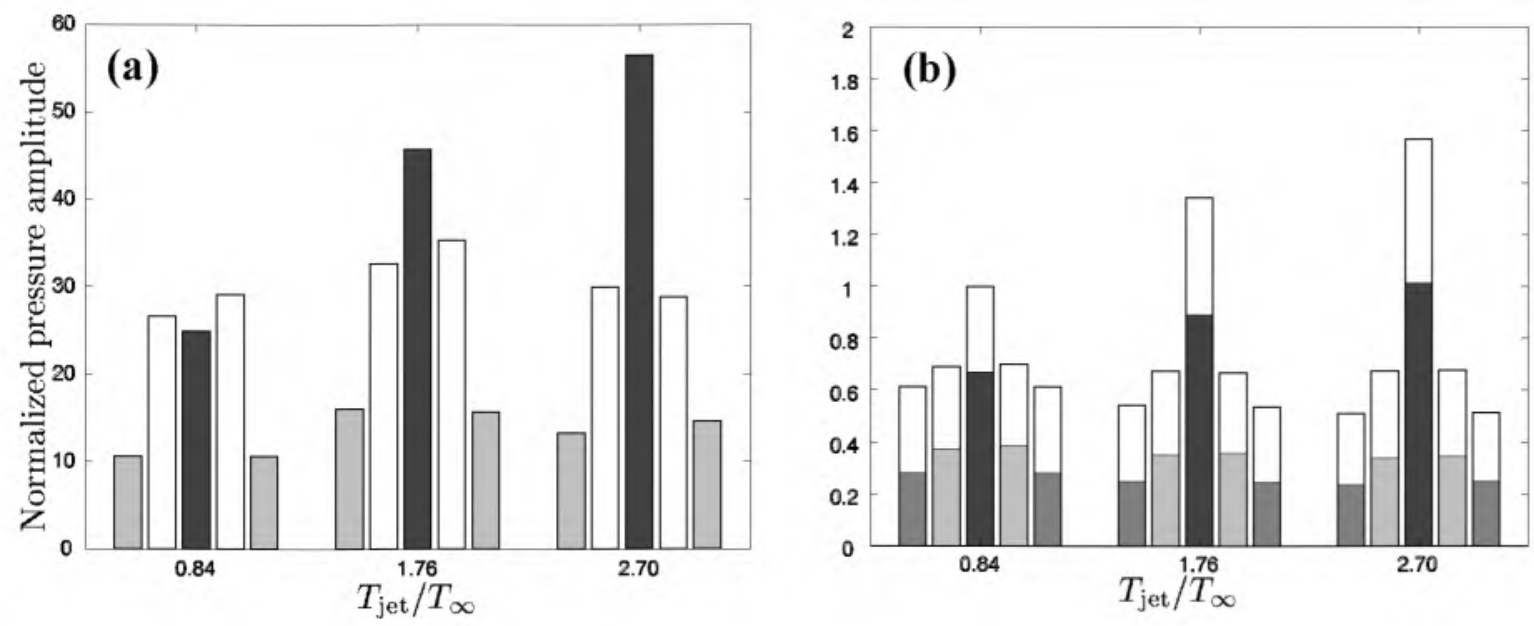

Figure 18. Comparison of the azimuthal mode balance at $M_{\infty}=0.9$ for $S t=0.30$. Notation is the same as figure 14.

\section{Conclusions}

Using near-field and mid-field phased-microphone arrays, we have measured the azimuthal-mode balance of instability waves and directivity of acoustic waves for the axisymmetric and first two azimuthal modes; consequently, we have compared these results to investigate the role of instability waves on low-frequency noise generation in a subsonic jet. To infer the instability-wave amplitude, we have applied a beam-forming technique developed in our previous study. ${ }^{19}$ For the acoustic waves, we have analyzed both overall pressure amplitude and amplitude of the primary POD mode of the cross spectral matrix. In this study, we have focused on three effects - compressibility, jet temperature, and chevron nozzles. These results are summarized in the following.

As the Mach number is increased for unheated jets, the growth rates of all the instability waves are suppressed, as predicted by linear stability analysis; however, their azimuthal-mode balances are almost unchanged. On the other hand, the suppression owing to compressibility is not observed in acoustic waves. More importantly, the sound radiation patterns are changed from the quadrupole-type radiation to highly directive patterns with increasing Mach number (the transition occurs around at $M_{\infty}=0.7$ ). The azimuthalmode balances of coherent sound at higher Mach numbers appear to be more similar to those of instability waves, and incoherent sound seems independent of the Mach number. This may imply that the noisegeneration mechanism of a high subsonic jet is related to instability waves.

We have studied the temperature dependence at two different Mach numbers $\left(M_{\infty}=0.5\right.$ and 0.9$)$. At the lower Mach number, the sound radiation patterns, particularly coherent sound, of the axisymmetric $(m=0)$ and the first azimuthal mode $(m=1)$ seem to change from the quadrupole-types to the dipole-types as the jet temperature increases. In contrast, at the higher Mach number, the trends of acoustic-wave amplification with temperature seem to follow the growth rates of instability waves. These phenomena may be related to the acoustic-source terms and turbulent fluctuations in the following manner. For unheated jets, jet noise is primarily generated by velocity fluctuations, producing the quadrupole-type radiation at low Mach numbers. As the jet is heated, the source term associated with density fluctuations generate dipole sources. At higher Mach numbers, however, the sound associated with velocity fluctuations still exceeds that generated by density fluctuations even for the heated jets.

The results of the chevron nozzles have demonstrated that the amplitude of instability waves is substantially enhanced by the chevron nozzles, while there is little change in the acoustic field. Thus, the noise reduction mechanisms with chevron nozzles are less likely related to the suppression of instability waves at 

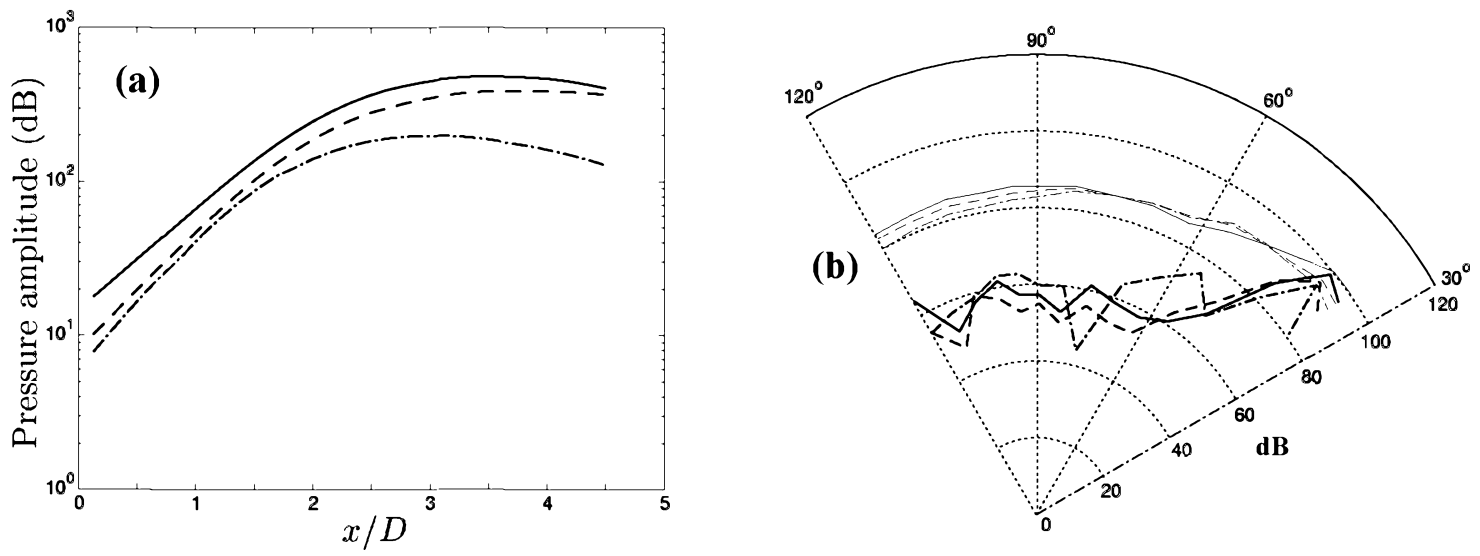

Figure 19. Streamwise evolution of eigenfunctions and radiation patterns of acoustic waves for SMC000 $\left(M_{\infty}=0.9\right.$ and $S t=0.35$, unheated jets). Notation is the same as figure 6. (a) Pressure amplitudes inferred from the beam-forming (unit is pascal). (b) Pressure amplitudes (thinner lines) and the first eigenmodes of the cross spectral matrices (thicker lines).
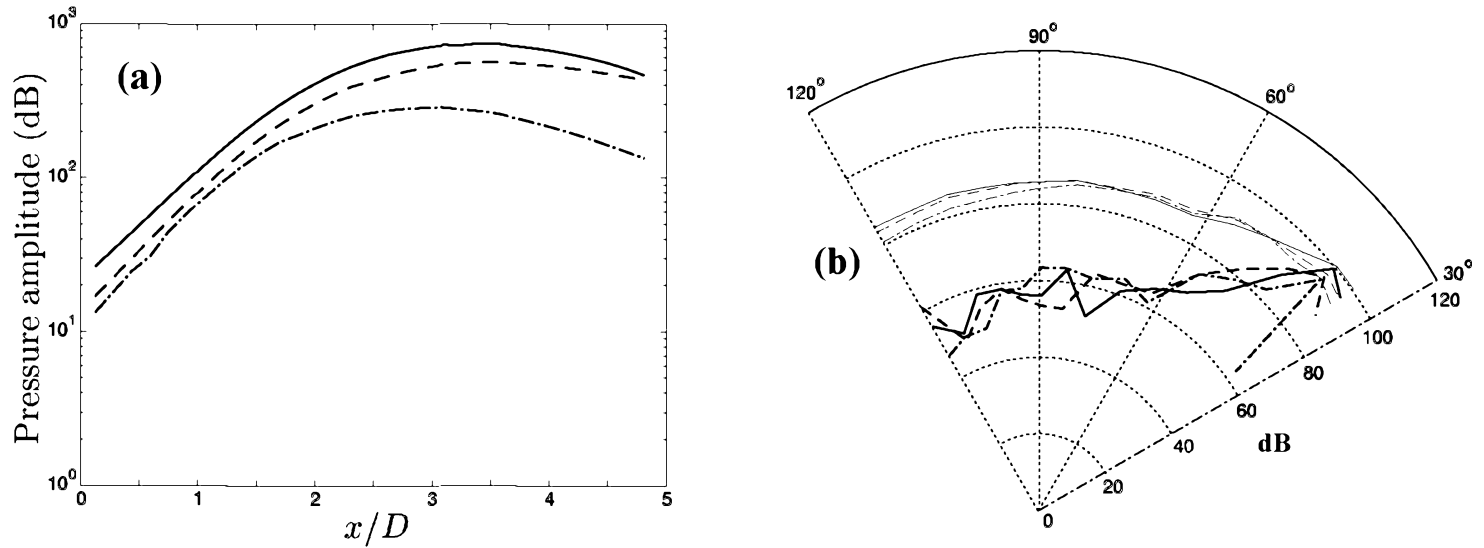

Figure 20. Streamwise evolution of eigenfunctions and radiation patterns of acoustic waves for SMC005. The flow conditions are the same as figure 19, and notation is the same as figure 6.

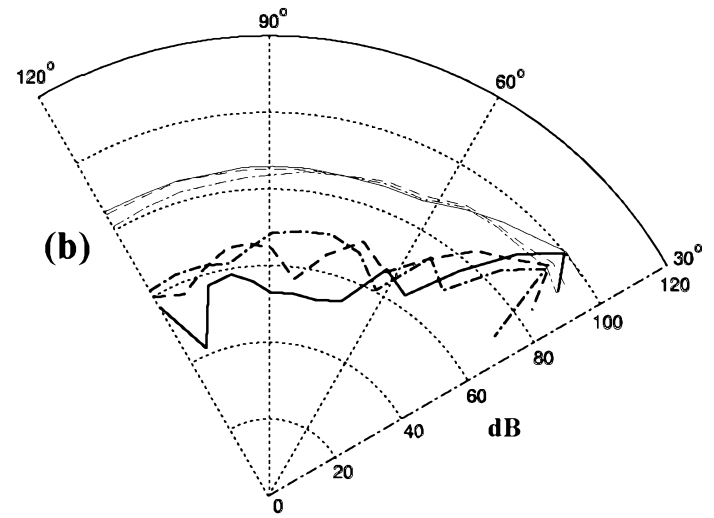

Figure 21. Radiation patterns of acoustic waves for SMC001. The flow conditions are the same as figure 19, and notation is the same as figure 6. Since the beam-forming algorithm is not applied due to the mean-flow distortion, the instability-wave evolution is not shown.

14 of 16

American Institute of Aeronautics and Astronautics 

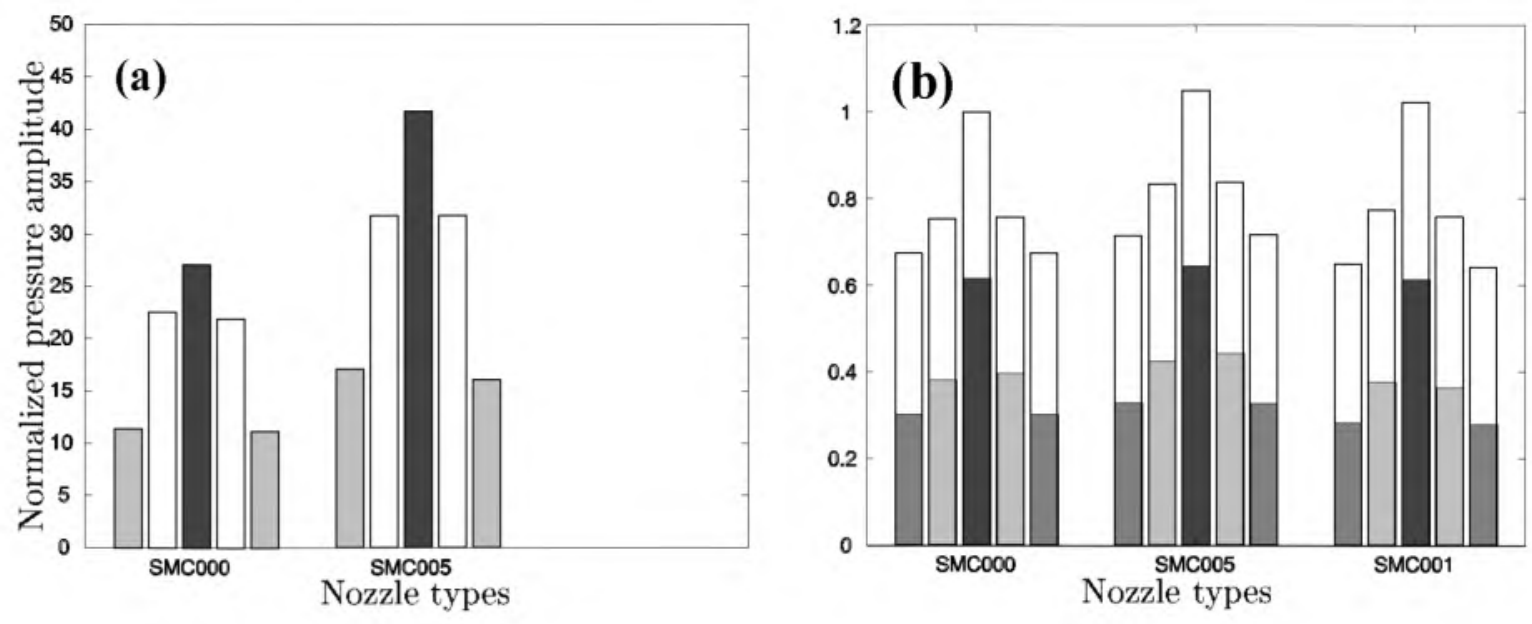

Figure 22. Comparison of the azimuthal mode balance for different nozzles $\left(M_{\infty}=0.9\right.$ and $S t=0.35$, unheated jets). Each cluster shows amplitudes of the $m=-2,-1,0,1,2$ modes from left to right. (a) Instability-wave balance (the peak amplitude along $r / D=0.5$ ). Amplitudes are normalized by that of $m=0$ at the nozzle exit. (b) Acoustic mode balance. White bars denote the overall sound, and colored ones denote the first POD modes. Amplitudes are normalized by the overall sound of SMC000 for $m=0$.

the nozzle exit. Hence, further investigation is necessary to understand the effects of chevron nozzles on the noise generation.

It should be reminded that the amplitude of instability waves has been determined using all microphones with eigenfunctions, while the acoustic radiation patterns have been calculated based the amplitude that is Fourier-transformed only at each ring. Moreover, the aliasing errors from higher azimuthal modes are more severe in acoustic waves; especially, the downstream five rings have only four microphones in the azimuthal direction. Therefore, we must be careful to interpret the results of radiation patterns.

Although we may need more microphones to precisely resolve the lobe patterns over a wide range of polar angles, this study appears to capture key transient features of jet noise - (i) from quadrupole to dipole patterns with increasing jet temperature at lower Mach numbers, and (ii) from quadrupole to highly directive patterns with increasing Mach number for unheated jets. At higher Mach numbers, however, an important question has remained. While more acoustic energy can be propagated downstream by the refraction effect with increasing Mach number, sound generated by instability waves is also considered to dominate the radiation at shallow angles. This point must be clarified in future study to understand the relation between the instability waves and the low-frequency jet noise.

\section{Acknowledgments}

The authors acknowledge the support of an AeroAcoustics Research Consortium (AARC) grant from the Ohio Aerospace Institute (OAI). We would like to express our deepest appreciation to Drs. J. Bridges, S.-S. Lee, and their colleagues at NASA Glenn Research Center for conducting all the experiments and fruitful discussions.

\section{References}

${ }^{1}$ Samimy, M., Zaman, K. B. M. Q., and Reeder, M. F., "Effect of tabs on the flow and noise field of an axisymmetric jet," AlAA J., Vol. 31, No. 4, 1993, pp. 609-619.

${ }^{2}$ Tam, C. K. W. and Zaman, K. B. M. Q., "Subsonic jet noise from nonaxisymmetric and tabbed nozzles," $A 1 A A J .$, Vol. 28, No. 4, 2000, pp. 592-599. 
${ }^{3}$ Simonich, J. C., Narayanan, S., Barber, T. J., and Nishimura, M., "Aeroacoustic characterization, noise reduction, and dimensional scaling effects of high subsonic jets," AIAA J., Vol. 39, No. 11, 2001, pp. $2062 \ldots 2069$.

${ }^{4}$ Callender, B. and Gutmark, E., "A Near field investigation of cherron nozzle mechanisms," AIAA paper 2003 3210, 2003.

${ }^{5}$ Butler, G. W. and Calkins, F. T., "Initial attempts to suppress jet noise using piezoelectric actuators," AIAA paper 2003-3192, 2003.

${ }^{6}$ Arakeri, V. H., Krothapalli, A., Siddavaram, V., and Alkislar, M. B., "On the use of microjets to suppress turbulence in a Mach 0.9 axisymmetric jet," J. Fluid Mech., Vol. 490, 2003, pp. 7598.

${ }^{7}$ Liu, J. C. T., "Developing large-scale wavelike eddies and the near jet noise field," J. Fluid Mech., Vol. 62, 1974, pp. $437-161$.

${ }^{8}$ Crighton, D. G. and Gaster, M., "Stability of slowly diverging jet flow," J. Fluid Mech., Vol. 77, 1976, pp. 397-413.

${ }^{9}$ Mankbadi, R. R. and Liu, J. T. C.., "A study of the interactions between large-scale coherent structures and fine-grained turbulence in a round jet," Proc. R. Soc. Lond. A, Vol. 298, 1981, pp. 541-602.

${ }^{10}$ Morris, P. J., Giridharan, M. G., and Lilley, G. M., "On the turbulent mixing of compressible free shear layers," Proc. R. Soc. Lond. A, Vol. 131, 1990, pp. 219213.

${ }^{11}$ Tam, C. K. W. and Morris, P. J., "The radiation of sound by the instability waves of a compressible plane turbulent, shear layer," J. Fluid Mech., Vol. 98, 1980, pp. 319381.

${ }^{12}$ Mankbadi, R. R., "On the interaction between fundamental and subharmonic instability waves in a turbulent round jet," J. Fluid Mech., Vol. 160, 1985, pp. 385-419.

${ }^{13}$ Crighton, D. G. and Huerre, P., "Shear-layer pressure fluctuations and superdirective acoustic sources," J. Fluid Mech., Vol. 220, 1990, pp. 355 368 .

${ }^{14}$ Colonius, T., Lele, S. K., and Moin, P., "Sound generated in a mixing layer," J. Fluid Mech., Vol. 330, 1997, pp. 375-109.

${ }^{15}$ Zaman, K. B. M. Q. and Hussain, A. K. M. F., "Vortex pairing in a circular jet under controlled excitation. Part 1. General jet response," J. Fluid Mech., Vol. 101, 1980, pp. 449-491.

${ }^{16}$ Tanna, H. K. and Ahuja, K. K., "A research program at Lockheed on tone excited jets -. Part I: Introduction," J. Sound Vib., Vol. 102, No. 1, 1985, pp. 57 61.

${ }^{17}$ Tam, C. K. W. and Morris, P. J., "Tone excited jets - Part V: A theoretical-model and comparison with experiment," J. Sourd Vib., Vol. 102, No. 1, 1985, pp. 119-151.

${ }^{18}$ Arndt, R. E. A., Long, D. F., and Glauser, M. N., "The proper orthogonal decomposition of pressure fluctuations surrounding a turbulent jet," J. Fluid Mech., Vol. 310, 1997, pp. 1-33.

${ }^{19}$ Suzuki, T. and Colonius, T., "Instability waves in a subsonic round jet detected using a near-field phased microphone array," 2006, accepted for J. Fluid Mech.

${ }^{20}$ Ghosh, A., Bridges, J., and Hussain, F., "Instantaneous directivity in jet noise by multipole decomposition," Trans. ASME: J. Vib. Acoust., Vol. 117, 1995, pp. 172 179.

${ }^{21}$ Fisher, M. J., Harper-Bourne, M., and Glegg, S. A. L., "Jet engine noise source location," J. Sound Vib., Vol. 58, No. 1, 1978 , pp. 117-126.

${ }^{22}$ Dougherty, R. P., "Phased array beamforming for aeroacoustics," AIAA professional development short course, 1999.

${ }^{23}$ Suzuki, T. and Butler, G. W., "New beam-forming algorithm for high speed jet flows," AIAA paper 2002-2505, 2002.

${ }^{24}$ Venkatesh, S. R., Polak, D. R., and Narayanan, S., "Beamforming algorithm for distributed source localization and its application to jet noise," AIAA J., Vol. 11, No. 7, 2003, pp. 1238-1216.

${ }^{25}$ Armstrong, R. R. and Michalke, A., "Coherent structures in jet turbulence and noise," AlAA J., Vol. 15, No. 7, 1977, pp. 1011-1017.

${ }^{26}$ Fuchs, H. V. and Michel, U., "Experimental evidence of turbulent source coherence affecting jet noise," AIAA J., Vol. 16, No. 9, 1978, pp. 871-872.

${ }^{27}$ Bridges, J. and Wernet, M., "Measurements of the aeroacoustic sound source in hot jets," AIA A paper $20033130,2003$.

${ }^{28}$ Bridges, J. and Brown, C., "Parametric testing of cherrons on single flow hot jets," AIAA paper 2001-2024, 2004.

${ }^{29}$ Suzuki, T., "Identification of multipole noise sources in low Mach number jets near the peak frequency," 2005, in revision for J. Acoust. Soc. Am.

${ }^{30}$ Tanna, H. K., "Experimental-study of jet noise: 1. Turbulent mixing noise," J. Sound Vib., Vol. 50, No. 3, 1977, pp. 105128.

${ }^{31}$ Lighthill, M. J., "On sound generated aerodynamically. 1. General theory," Proc. R. Soc. Lond. A, Vol. 211, 1952, pp. $561-587$.

${ }^{32}$ Lilley, "On the noise from jets," AGARD-CP-131, 1971.

${ }^{33}$ Tester, B. J. and Morfey, C. L., "Developments in jet noise modelling-theoretical predictions and comparisons with measured data," J. Sound Vib., Vol. 46, No. 1, 1976, pp. 79103.

16 of 16

American Institute of Aeronautics and Astronautics 